\title{
Editorial review board
}

The 13 chapters in this book have been brought to publication with the help of an editorial review board dedicated to peer review. The 20 members of the board are committed to the field of environmental taxation and are active participants in environmental taxation events around the world:

\section{Chair:}

Natalie P. Stoianoff

University of Technology Sydney, Australia

\section{Members:}

Hope Ashiabor

Macquarie University, Australia

Patricia Blazey

Macquarie University, Australia

Bill Butcher

University of New South Wales, Australia

Jacqueline Cottrell

Green Budget Europe, Germany

Damien Giurco

University of Technology Sydney, Australia

Wayne Gumley

Monash University, Australia

Ann Hansford

University of Exeter, $U K$

Larry Kreiser

Cleveland State University, USA

David Leary

University of Technology Sydney, Australia 
Soocheol Lee

Meijo University, Japan

Roberta Mann

University of Oregon, USA

Janet E. Milne

Vermont Law School, USA

Anna Mortimore

Griffith University, Australia

Sven Rudolph

Kyoto University, Japan

Rahmat Tavalali

Walsh University, USA

Dodo J. Thampapillai

National University of Singapore, Singapore

Stefan Weishaar

University of Groningen, the Netherlands

Jian $\mathrm{Wu}$

Renmin University, China

Yan $\mathrm{Xu}$

The Chinese University of Hong Kong, Hong Kong 